\title{
Effect of Provisional Cements on Shear Bond Strength of Porcelain Laminate Veneers
}

\author{
Subutay Han Altintas ${ }^{a}$ \\ Onjen Tak ${ }^{\mathrm{b}}$ \\ Asli Secilmis ${ }^{c}$ \\ Aslihan Usumez ${ }^{\mathrm{d}}$
}

\section{ABSTRACT}

Objectives: The purpose of this study was to evaluate the effect of three provisional cements and two cleaning techniques on the final bond strength of porcelain laminate veneers.

Methods: The occlusal third of the crowns of forty molar teeth were sectioned and embedded in autopolymerizing acrylic resin. Dentin surfaces were polished and specimens were randomly divided into four groups $(n=10)$. Provisional restorations were fabricated and two provisional restorations were cemented onto each tooth. Restorations were fixed with one of three different provisional cements: eugenol-free provisional cement (Cavex), calcium hydroxide (Dycal), and light-cured provisional cement (Tempond Clear). Provisional restorations were removed with either a dental explorer and air-water spray, or a cleaning bur (Opticlean). In the control group, provisional restorations were not used on the surfaces of specimens. IPS Empress 2 ceramic discs were luted with a dual-cured resin cement (Panavia F). Shear bond strength was measured using a universal testing machine. Data were statistically analyzed by ANOVA, Tukey's HSD and Dunnett tests. Surfaces were examined by scanning electronic microscopy.

Results: Significant differences were found between the control group and both the light-cured provisional cement groups and the eugenol-free provisional cement-cleaning bur group $(P<.05)$. Groups that had received light-cured provisional cement showed the lowest bond strength values.

Conclusions: Selection of the provisional cement is an important factor in the ultimate bond strength of the final restoration. Calcium hydroxide provisional cement and cleaning with a dental explorer are advisable. (Eur J Dent 2011;5:373-379)

Key words: Provisional cement; Porcelain laminate veneer; Surface cleaning techniques.

a Asistant Professor, Karadeniz Technical University, Faculty of Dentistry, Department of Prosthodontics, Trabzon, Turkey.

b Assistant Professor, University of Kocaeli, Faculty of Dentistry, Department of Prosthodontics, Kocaeli, Turkey.

c Assistant Professor, University of Gaziantep, Faculty of Dentistry, Department of Prosthodontics, Gaziantep, Turkey. d Professor, University of Gaziantep, Faculty of Dentistry, Department of Prosthodontics, Gaziantep, Turkey.

- Corresponding author: Dr. Asli Secilmis Gaziantep Universitesi, Dishekimligi Fakultesi, Protetik Dis Tedavisi A.D., Gaziantep, Turkey. Phone: $+903423606060-76611$

Fax: +90 3423610610

E-mail: acarasliahotmail.com 


\section{INTRODUCTION}

Porcelain laminate veneers (PLVs) have been employed successfully in clinical practice since 1983 to mask teeth with intrinsic staining attributed to ageing, tetracycline, or fluorosis. The clinical success of PLVs is attributed to the intimate bond achieved between the restoration and tooth structure through the luting cement. ${ }^{1}$

Indirect restorations usually require temporization for the protection of the pulp and to restore the patient's aesthetic and functional needs. A provisional cement is used to fix this type of temporary restoration onto the tooth. ${ }^{2}$

Regardless whether a conventional or luting cement is used, proper cleaning of the abutment teeth is important to avoid any interference along the interface between the abutment surface and the luting cement. ${ }^{3}$ The polymerization of composite resins and dentin bonding agents are induced by radicals, which are initiated either chemically or by light. ${ }^{2}$ Residual provisional cement and debris might therefore impair the etching quality of the tooth surface, the infiltration of the adhesive system, or may even inhibit the polymerization of the resinous monomers and thus the fit and final bonding of the PVL. 4,5

Several investigators have studied methods for the removal of provisional cement in vitro. ${ }^{3,5-9}$ Button et $\mathrm{al}^{7}$ reported that higher retentive strengths for glass ionomer and zinc polycarboxylate cements were obtained with tooth preparations cleansed with plain flour pumice than those cleansed with an explorer only. Terata ${ }^{9}$ showed that the removal of provisional cement from bovine enamel and dentin with an explorer was incomplete. Bachmann et $\mathrm{al}^{6}$ investigated the bond strength of dentin bonding agents after teeth were cleansed with a scaler, a cotton pellet with pumice, and different soaps. All of the soaps tested decreased shear bond strength values, thus, the use of soap was not recommended for clinical removal of remnants of provisional cements prior to adhesive cementation. Kanakuri et al ${ }^{10}$ reported that the use of a rotational brush with running water was the best method. For removal of debris and remnants from the dentin surface, different cleaning agents containing ethanol, ethyl acetate, acetone, or chlorhexidine digluconate have been marketed. The water-miscible solvents, such as acetone or ethanol, have been used in bonding agents and are thought to behave as a water chaser and to facilitate resin monomer penetration into the collagen network. ${ }^{11-13}$

The purpose of this in vitro study was to evaluate the effect of three provisional cements and two cleaning techniques on the final bond strength of PLVs. The bonding interfaces of the final restorations are also examined by scanning electron microscopy (SEM). The working hypothesis was that a dentin surface cleaned with a cleaning bur will show higher bond strength values than a dentin surface cleaned with a dental explorer and airwater spray.

\section{MATERIALS AND METHODS}

Tooth preparation

Forty non-carious lower wisdom molars were cleaned and stored in distilled water at room temperature immediately after extraction. The occlusal thirds of the crowns were sectioned with a water-cooled slow-speed diamond saw sectioning machine (Isomet, Buehler Ltd., Lake Bluff, IL, USA). The teeth were fixed into an autopolymerizing acrylic resin (Meliodent, Bayer Dental Ltd., Newbury, UK), with the ground surface upward and parallel to the support. Dentin surfaces were polished with 600 and 800 grit $\mathrm{SiC}$ for 30 seconds to standardize the smear layer. All specimens were randomly divided into four groups of 10 teeth; three according to the provisional cement used and one for the control group.

The fabrication and cementation of the provisional restorations

Sixty provisional restorations $13 \mathrm{~mm}$ in diameter and $2 \mathrm{~mm}$ in height) were fabricated from autopolymerizing acrylic resin (Temdent, Schültz, Wiel Dental GmbH, Rosbach, Germanyl using silicone impressions as a matrix.

Two provisional restorations were cemented onto each tooth to evaluate the effect of different cleaning procedures. Provisional restorations were cemented under finger pressure. ${ }^{14}$ Provisional restorations were fixed with one of the three different provisional cements: eugenol-free provisional cement (Cavex, Holland BV, Haarlem, Holland), calcium hydroxide (Dycal, Kerr, Danbury, CT, USA), and light-cured provisional cement (Tempond Clear, GC, Alsip, IL, USA). In the 
light-cured provisinal cement group, a very small amount of light-cured provisional cement was applied to the provisional restorations. The provisional restorations were placed on the dentin surface and the cement was light-cured for 60 seconds. After removing excess cement, the specimens were stored in distilled water at $37^{\circ} \mathrm{C}$ for 1 week. In the control group, no provisional restorations were applied.

After storage, provisional restorations were dislodged and the surfaces were cleaned by the following procedures: The provisional cement on one half of the specimen was mechanically removed with a dental explorer (Kohler Medizintechnik GmbH \& Co., Neuhausen, Germanyl until the dentin surface was macroscopically clean, and then the dentin surface was throughly rinsed with an air-water spray. The other half of the dentin block were treated with a rotary instrument (W\&H Trend WD-58, W\&H Dentalwerk Bürmoos GmbH, Austrial and cleaning bur (Opticlean, Kerr, Danbury, CT, USAl for 1 minute.

Ceramic disc fabrication and final cementation

Wax sprues $3 \mathrm{~mm}$ in diameter were invested, heated, and pressed according to the manufacturer's instructions and 70 ceramic discs (IPS Empress, Ivoclar Vivadent, Schaan, Liechtenstein) were fabricated. After the heat-pressing procedure, the ceramic rods were cut into discs $3 \mathrm{~mm}$ in diameter and $2 \mathrm{~mm}$ in height. IPS Empress discs were divested by airborne-particle abrasion with $100 \mu \mathrm{m}$ aluminum oxide (Korox 100, Bego Bremer Goldschlaagerei Wilh. Herbst GmbH \& Co., Bremen, Germanyl. The air pressure for sandblasting was maintained at 1 bar.

Ceramic cylinders were etched with phosphoric acid gel (K Etchant, Kuraray Co., Ltd. Osaka, Japan) for 5 seconds. A layer of silane coupling agent combination (Clearfil Porcelain Bond Activator and Clearfil Liner Bond 2V Primer, Kuraray Co., Ltd., Osaka, Japan) was applied to the ceramic bonding surfaces for 5 seconds, and then air-dried. A self-etching primer (Panavia F ED, Kuraray Co., Ltd., Osaka, Japan) was applied to the dentin surface for 60 seconds and gently air-dried. Panavia $F$ universal and catalyst pastes were mixed for 20 seconds and applied to both the dentin surface and the bonding surface of the ceramic disc. Ceramic discs were placed on the dentin surface with light finger pressure as in clinical practice and excess cement was removed with an explorer. An oxygenblocking gel lOxyguard II, Kuraray Co. Ltd., Osaka, Japan) was applied for 3 minutes. Photo polymerization was performed with the light-polymerizing unit (Hilux 550, Express Dental Products, Toronto, Canadal at $550 \mathrm{~mW} / \mathrm{cm}^{2}$ for 40 seconds. The light tip of the curing unit was centered on the ceramic restoration without any distance. All procedures were performed by one operator. The specimens were kept at $37^{\circ} \mathrm{C}$ for 1 day before the shear bond test.

Shear bond strength tests and statistical analysis

Shear bond strength tests were performed with a universal testing machine (TSTM 02500, Elista Corp., Istanbul, Turkeyl. A knife-edge shearing rod at a crosshead speed of $0.5 \mathrm{~mm} / \mathrm{min}$ was used on the bonded interface until the bond failure occurred. Shear-bond force was recorded digitally with a personal computer. Shear bond strength was calculated according to the following formula and expressed in MPa: Stress= Failure Load (N) / Surface Area $\left(\mathrm{mm}^{2}\right)$

Analysis of variance (ANOVA) was used to analyze the data for significant differences. Tukey's honestly significant difference (HSD) test and Dunnett tests were used to perform multiple comparisons $(\alpha=.05)$. The shear bond strength values were analyzed with the SPSS 13 for Windows statistical software (SPSS PC, Vers.10.0, SPSS, Chicago, Ill).

\section{SEM analysis}

A new specimen belonging to each test group was prepared for SEM analysis. The procedures used to bond the surface were identical to those used for the other test procedures already described, except that the teeth were not fixed into the autopolymerizing acrylic resin. These specimens were used to evaluate the degree of resin tag penetration into the dentin surface during the bonding process.

After storage for 24 hours at $37^{\circ} \mathrm{C}$, specimens were sectioned bucculingually in a low-speed saw (Isomet, Buchler Ltd, IL, USA) with a diamond-rim blade. The bonding interfaces were first polished with 240, 400 and 600 grit $\mathrm{SiC}$ and then were etched with $15 \%$ phosphoric acid for 2 minutes. Specimens were washed and gently air-dried for 3 seconds, 
and then mounted on SEM stubs and sputtercoated with gold. Interfaces were observed with the SEM (JSM-5600, Joel Ltd., Tokyo, Japan) and photomicrographs with a magnification of $\times 1500$ 2000 were taken of different regions of the bonding interface (Figures 1-4).

\section{RESULTS}

Shear bond strength tests

One-way ANOVA test revealed statistically significant differences among the shear bond strengths $(P<.05, P=.000)$. Table 1 shows results of shear bond strength tests.

No statistically significant differences were found among cleaning procedures ( $P>.05)$, eugenol-free provisional cement, calcium hydroxide and light-cured provisional cement groups, $P=.44$, $\mathrm{P}=.26, \mathrm{P}=1.00$.

Significant differences were found between the control group and both the light-cured provisional cement groups and the eugenol-free provisional cement-cleaning bur group (Dunnett, $P<.05$ ). Ceramic discs placed on tooth surfaces that had received light-cured provisional cement showed the lowest bond strength values of all test groups.

\section{SEM analysis}

All specimens showed adhesive failures between the luting cement and dentin interface. None of the specimens showed mixed failure or cohesive failure within the ceramic discs, the luting cement, or dentin bond interfaces.

SEM examination showed that the bonding to dentin surface was better in the control group (Figure 1). Resin tags that stretched out from hybrid layer to the dentin were clear in the eugenol-free provisional cement and calcium hydroxide groups (Figures 2 and 3). In the light-cured provisional cement group, dentin-resin bonding area exhibited a disrupted interface. The dentinal tubules were covered by the remnants of the provisional cement on the dentin surfaces (Figure 4).

\section{DISCUSSION}

In the current study, three provisional cements and two cleaning techniques were evaluated for their effects on the final bond strengths of PLVs. The results obtained did not support the research hypothesis as no differences were found between the cleaning procedures.

Freshly cut dentin is the ideal substrate for dentin bonding. ${ }^{15}$ In practice, freshly cut dentin is present only at the time of tooth preparation, prior to taking impressions. Significant reductions in bond strength can occur when dentin is contaminated with various provisional cements, compared to freshly cut dentin. ${ }^{16}$ Since some period of time is necessary to make the final restoration, a provisional restoration is used for approximately 10 days after the fixation. This makes mechanical and/or chemical cleaning procedures necessary before the definitive restoration is cemented. In this current study, after a period of 1 week, provisional cements were found to affect the final bond strength to dentin, although the bond strength test achieved acceptable values for all cases. Kanakuri et $\mathrm{al}^{10}$ reported mean bond strength values varied between 12.7-7.6 MPa, which are similar to the results of the present study (11.8-5.9 MPa).

In the current study, the provisional cement type affected dentin adhesion regardless of the different cleaning procedures. Among the provisional cements used in our study, the calcium hydroxide cement seems to promote higher bond strength than the other cements. Contrary to the results of our study, Fonseca et $\mathrm{l}^{5}$ reported that the calcium hydroxide cement had a lower bond strength than zinc oxide eugenol and zinc oxide eugenol-free provisional cements.

Grasso et $\mathrm{al}^{3}$ stated that abutment teeth cleansed with a prophy cup and flour pumice exhibited the least amount of residual provisional cement compared to explorer and air-water spray or cotton pellet and chlorhexadine gluconate. In the current study, higher bond strength values

Table 1. Shear bond strength values (MPa).

\begin{tabular}{lcc}
\hline & Dental explorer & Cleaning bur \\
\hline Cavex (Eugenol-free provisional cement) & $10 \pm 1.5 \mathrm{bc}$ & $8.2 \pm 1.5 \mathrm{ab}$ \\
Dycal (Calcium hydroxide provisional cement) & $11.7 \pm 2.3 \mathrm{c}$ & $9.7 \pm 2.1 \mathrm{bc}$ \\
Tempond Clear (Light-cured provisional cement) & $6.2 \pm 2 \mathrm{a}$ & $5.9 \pm 2.4 \mathrm{a}$ \\
Control (No provisional cement) & $11.8 \pm 1.6 \mathrm{c}$ & \\
\hline * Same letters were not significantly different at $P<.05$. & &
\end{tabular}

* Same letters were not significantly different at $\mathrm{P}<.05$. 


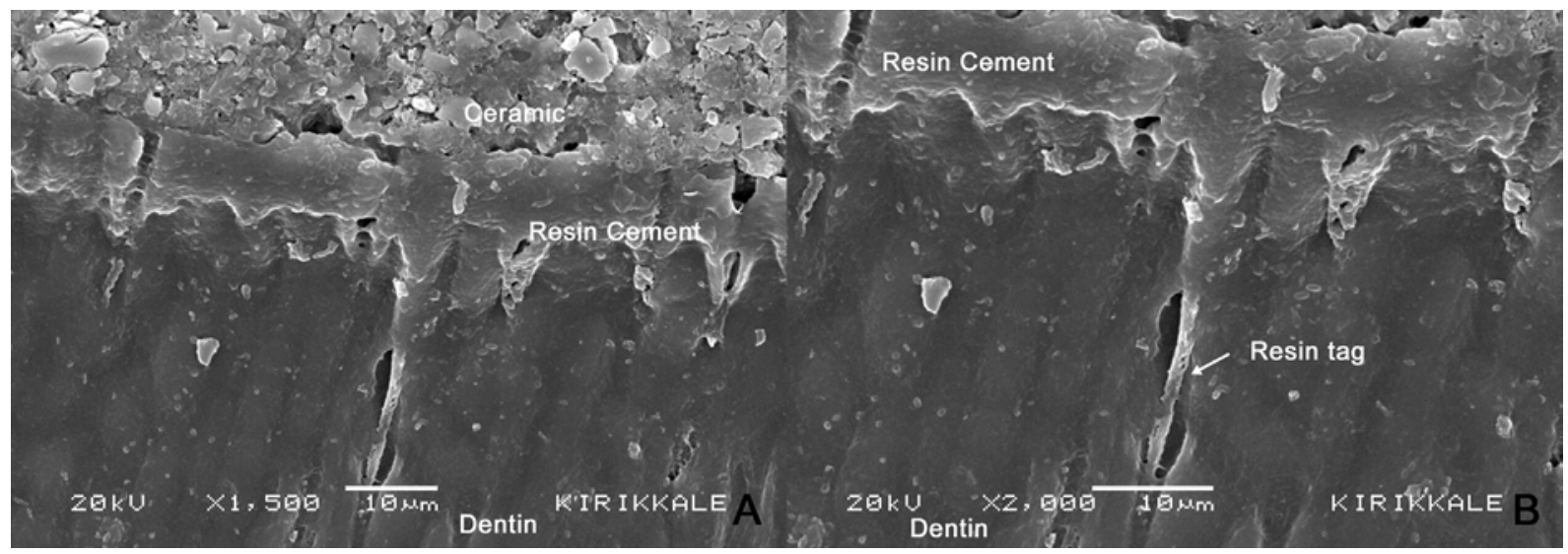

Figure 1. SEM views of adhesive-dentin interface of control group (no provisional restoration); A: ×1500, B: 2000.

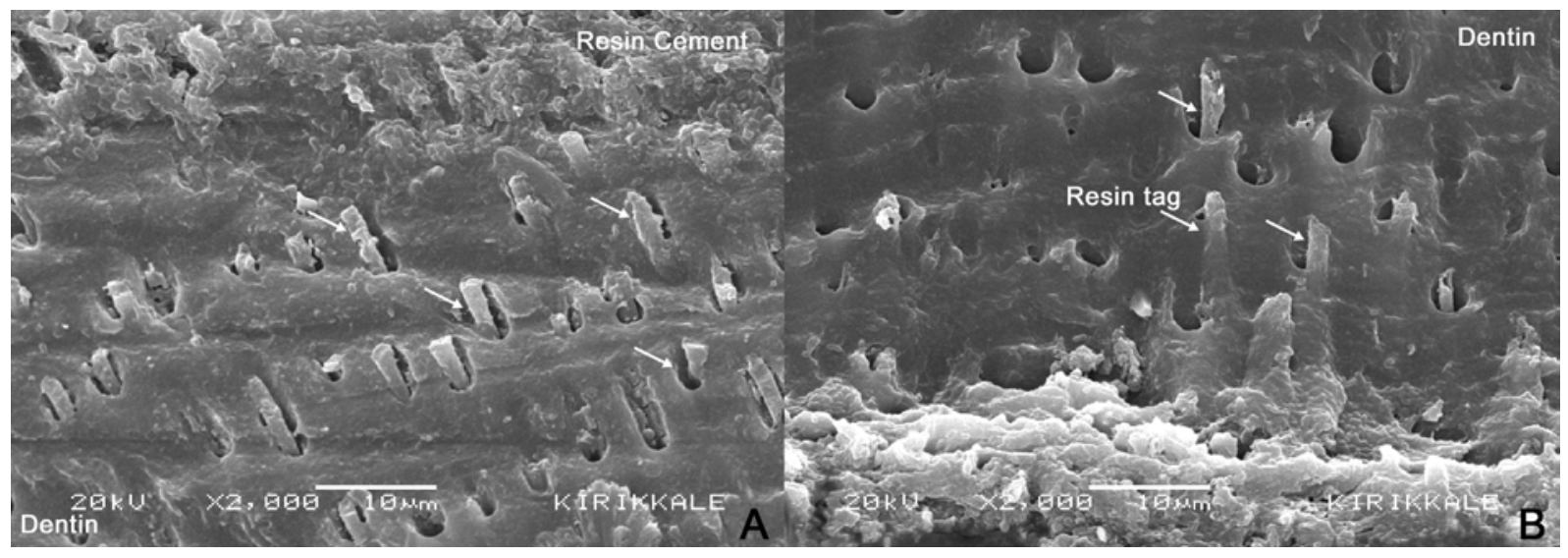

Figure 2. SEM views of adhesive-dentin interface of the eugenol-free provisional cement groups. A: Dental explorer and air-water spray $\times 2000$; B: Cleaning bur $\times 2000$.

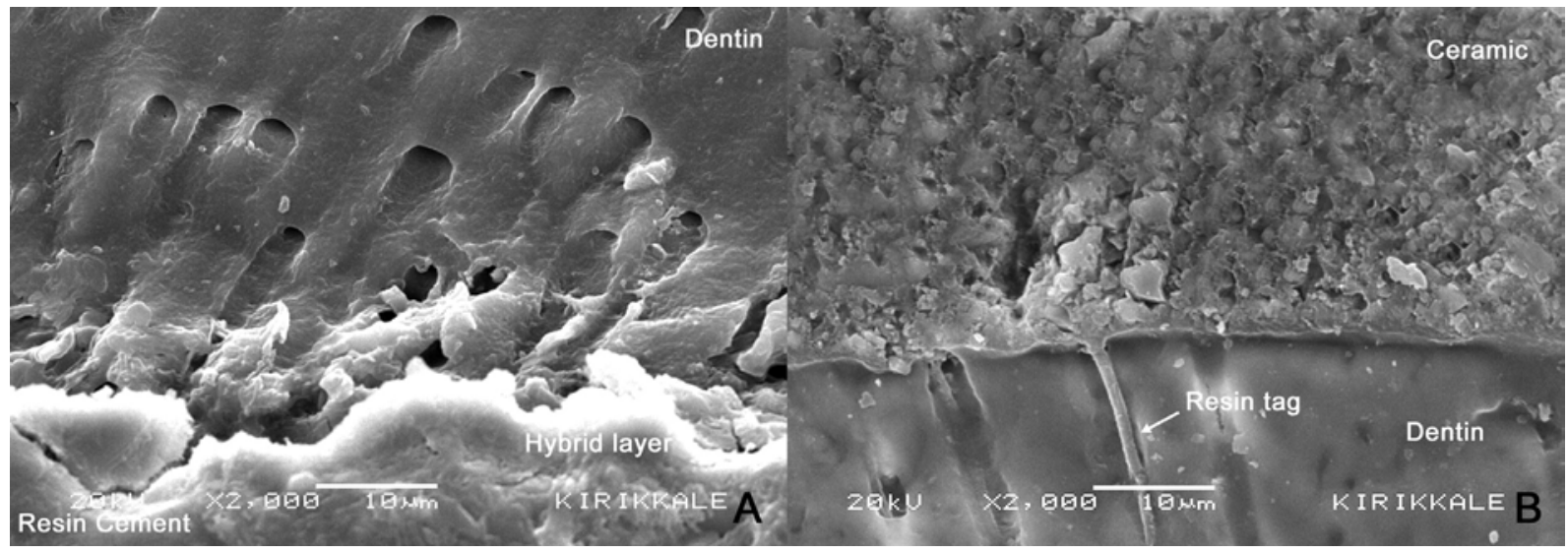

Figure 3. SEM views of adhesive-dentin interface of the calcium hydroxide provisional cement groups. A: Dental explorer and air-water spray $\times 2000$; B: Cleaning bur $\times 2000$.

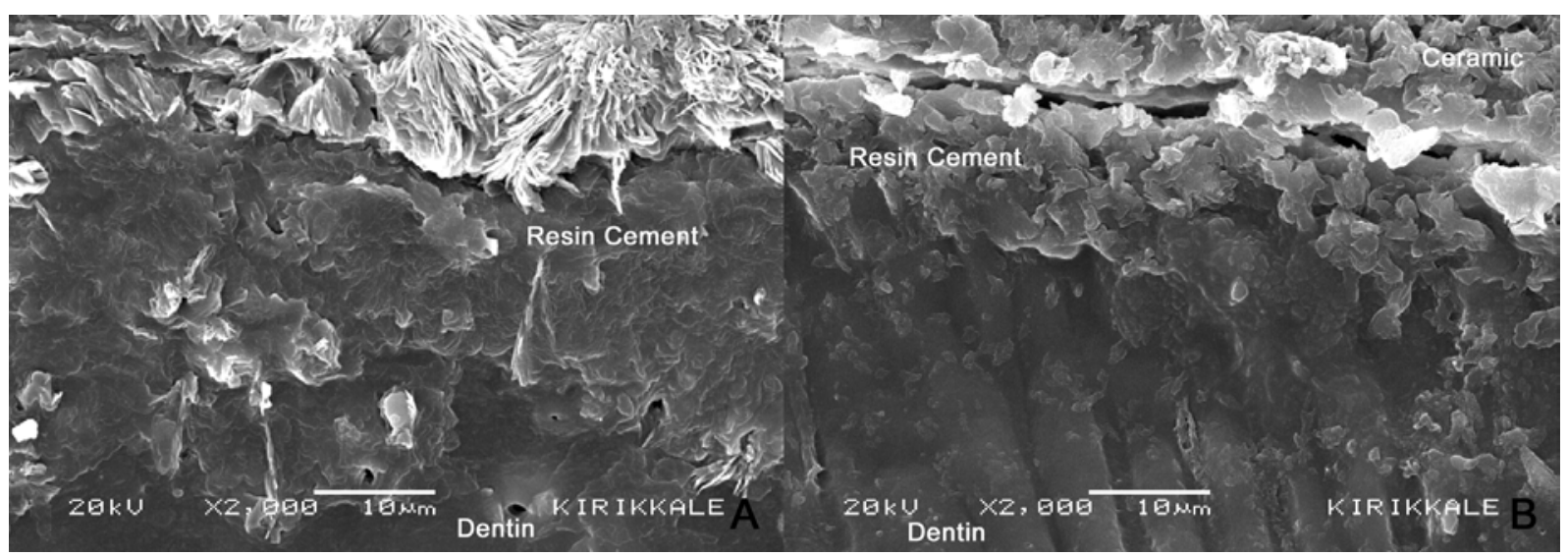

Figure 4. SEM views of adhesive-dentin interface of the light-cured provisional cement groups. A: Dental explorer and air-water spray $\times 2000$; B: Cleaning bur $\times 2000$. 
were achieved when a dental explorer and airwater spray was used as the cleaning procedure. In all groups, more effective removal of residual provisional cement was obtained by cleaning the dentin surface with a dental explorer and air-water spray than with a cleaning bur. Sarac et $\mathrm{al}^{8}$ reported that the provisional cement could plug the dentinal tubules into which the resin luting agent penetrates and the lowest bond strength was obtained with a rotary instrument and a cleaning bur. In the current study, specimens exhibited adhesive type failures in all groups, with the fracture occurring through the luting cement-dentin interface. A view of the control group is presented in Figure 1 , which shows the resin that penetrated into the dentin. Resin tags within the dentin tissue were clear and long for the eugenol-free provisional cement and calcium hydroxide provisional cement groups, whereas they were absent for light-cured provisional cement group. This fact supports data that the reduced bond strength of luting cement to tooth surfaces resulted from the presence of provisional cement residues that were not completely removed before permanent cementation.

Specimens for testing were prepared using human teeth. The manufacturer's instructions were followed carefully when PLVs were prepared to ensure that in vitro procedures were the same as those used clinically, but in the current study, neither thermal cycling nor mechanical stress was applied. In a previously published study, Paul and Shärer ${ }^{17}$ showed that considerable differences for the bond strength values could be obtained if dentin bonding agents were applied to a dry dentin surface vs. a dentin surface that was constantly kept under intrapulpal pressure. In the current study, materials were applied to dentin that was not under intrapulpal pressure. These factors may limit the direct application of the study results to in vivo situations. Moreover, only one type of dual polymerizing luting cement and one-step/selfetch adhesive system were used. Different results may be found with different luting cements or with total etch adhesive systems.

\section{CONCLUSIONS}

In the present study, significant differences were apparent in provisional cement groups, with light cured provisional cement groups having the lowest shear bond strength values. As the perma- nent bonding of PLVs can be adversely affected, the provisional cement and cleaning technique should be carefully selected. Calcium hydroxide provisional cement and cleaning with a dental explorer are suggested, since, within the limitations of current study, this technique yielded a shear bond strength value nearest to that of the control group.

\section{REFERENCES}

1. McLean JW. Ceramics in clinical dentistry. Br Dent $J$ 1988;164:187-194.

2. Hansen EK, Asmussen E. Influence of temporary filling materials on effect of dentin-bonding agents. Scand J Dent Res 1987;95:516-520.

3. Grasso CA, Caluori DM, Goldstein GR, Hittelman E. In vivo evaluation of three cleaning techniques for prepared abutment teeth. J Prosthet Dent 2002;88:437-441.

4. Dumfahrt H, Göbel $G$. Bonding porcelain laminate veneer provisional restorations: An experimental study. J Prosthet Dent 1999;82:281-285.

5. Fonseca RB, Martins LRM, Quagliatto PS, Soares CJ. Influence of provisional cements on ultimate bond strength of indirect composite restorations to dentin. J Adhes Dent 2005; 7:225-230.

6. Bachmann M, Paul SJ, Lüthy H, Schärer P. Effect of cleaning dentine with soap and pumice on shear bond strength of dentine-bonding agents. J Oral Rehabil 1997;24:433-438.

7. Button GL, Moon PC, Barnes RF, Gunsolley JC. Effect of preparation cleaning procedures on crown retention. $J$ Prosthet Dent 1988;59:145-148.

8. Sarac D, Sarac YS, Kulunk S, Kulunk T. Effect of the dentin cleaning techniques on dentin wetting and on the bond strength of a resin luting agent. J Prosthet Dent 2005;94:363369.

9. Terata R. Characterization of enamel and dentin surfaces after removal of temporary cement-study on removal of temporary cement. Dent Mater J 1993;12:18-28.

10. Kanakuri K, Kawamoto Y, Matsumura H. Influence of temporary cement remnant and surface cleaning method on bond strength to dentin of a composite luting system. $J$ Oral Sci 2005;47:9-13.

11. Finger WJ, Balkenhol M. Rewetting strategies for bonding to dry dentin with an acetone-based adhesive. J Adhes Dent 2000;2:51-56.

12. Nakaoki Y, Nikaido T, Burrow MF, Tagami J. Effect of residual water on dentin bond strength and hybridization of a one-bottle adhesive system. Oper Dent 2002;27:563-568. 
13. Pereira GD, Paulillo LA, De Goes MF, Dias CT. How wet should dentin be? Comparison of methods to remove excess water during moist bonding. J Adhes Dent 2001;3:257264.

14. Castelnuovo J, Tjan AHL, Phillips K, Nicholls JI, Kois JC. Fracture load and mode of failure of ceramic veneers with different preparations. J Prosthet Dent 2000;83:171-180.

15. Cagidiaco MC, Ferrari M, Garberoglio R, Davidson CL. Dentin contamination protection after mechanical preparation for veneering. Am J Dent 1996;9:57-60.

16. Magne P, Kim TH, Cascione D, Donovan TE. Immediate dentin sealing improves bond strength of indirect restorations. J Prosthet Dent 2005;94:511-519.

17. Paul SJ, Schärer P. Intrapulpal pressure and thermal cycling: Effect on shear bond strength of eleven modern dentin bonding agents. $J$ Esthet Dent 1993;4:179-185. 